\section{Familial autosomal dominant dopa responsive Parkinson's disease in three living generations showing extreme anticipation and childhood onset}

\author{
P J Morrison, R B Godwin-Austen, J A Raeburn
}

\begin{abstract}
We describe a three generation family with Parkinson's disease showing autosomal dominant inheritance with extreme anticipation. Familial Parkinson's disease in three living generations is extremely rare, and anticipation is an unusual and interesting feature. Anticipation was shown in all generations and may have involved previous generations. Some cases of familial Parkinson's disease may therefore involve a trinucleotide repeat gene as part of the causal mechanism.

( $f$ Med Genet 1996;33:504-506)
\end{abstract}

Key words: Parkinson's disease; anticipation; autosomal dominant.

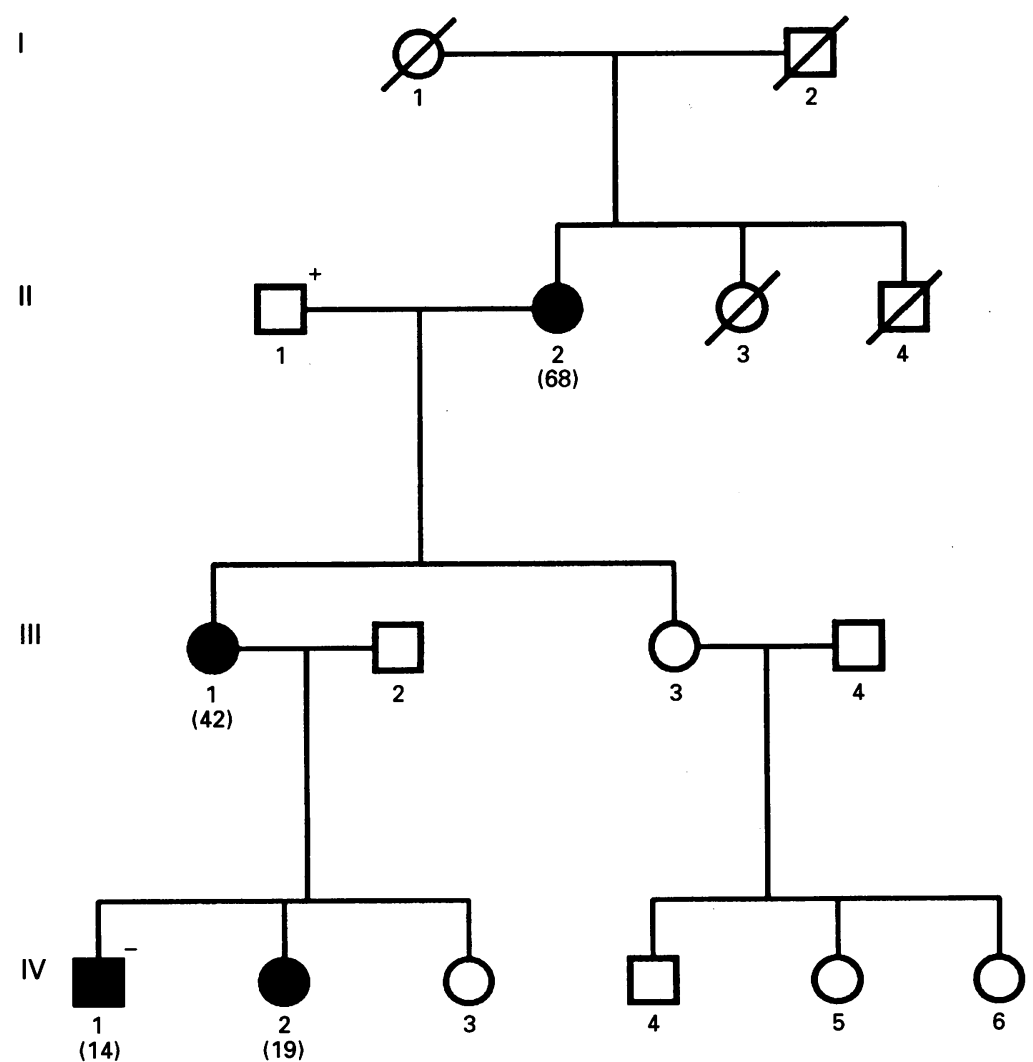

Pedigree of family showing index case and living affected members. There is no history of other cases of Parkinson's disease or other related disorders in generation I. Affected cases are shown as full shading. The age of onset is indicated in brackets for affected cases. indicates Klinefelter's syndrome, + indicates coincidental arteriosclerotic Parkinsonism.
There is now increasing evidence of a genetic component in the pathogenesis of Parkinson's disease (PD). ${ }^{1}$ Several families have been described with autosomal dominant inheritance. ${ }^{23}$ Most cases are two generation families, although three generation families or rarely even larger multigeneration families have been described. ${ }^{24}$ Although previous families described with "Parkinson's disease" had additional unusual features and are probably not true PD, the few recently documented cases comply with criteria now established by Maranganore $e t a l^{2}$ requiring people to have evidence of at least two of dystonia, tremor, rigidity, and bradykinesia; L-dopa responsiveness; and the presence of unilateral or asymmetrical symptoms or signs at onset. Anticipation, increasing severity or earlier age of onset of a condition as it is transmitted through generations, is a feature that has been postulated to occur in $\mathrm{PD},{ }^{5}$ but in practice has rarely been described. ${ }^{6-8}$ Since anticipation occurs in trinucleotide repeat disorders such as Huntington's disease, myotonic dystrophy, or possibly even schizophrenia, ${ }^{9-11}$ its occurrence in Parkinson's disease could be indicative of a similar trinucleotide repeat mechanism. We describe a family conforming to the above clinical criteria, but with anticipation as an additional feature, causing a change of onset in one patient from 68 years to early childhood $P D$ in a boy at 14 years.

Case reports (figure)

Patient II 2 , the index case, was ascertained in 1983 at the age of 71 years with an onset of symptoms in her late $60 \mathrm{~s}$. Her presenting sign was tremor of her right hand. She had generalised hypokinesia with slight postural change, but no rigidity. Her symptoms were dopa responsive (Sinemet). At her current age of 82 , she has increased difficulty with walking and a little rigidity.

Patient III $\cdot 1$, her daughter, initially noticed tremor of her left arm at the age of 42 . At 48 , she developed dysarthria and difficulty with salivation. She was dopa responsive (Sinemet, Pergolide), and also described akathisia relieved by Pergolide. On treatment, her only 
signs are slight slowness of movement in her right hand, with minimal rigidity.

Patient IV 1 was diagnosed at the age of 14 when he developed a right handed tremor. This now affects both hands and he has associated paucity of fine hand movements with a little rigidity, and marked dysarthria. He has mental retardation (MR) and a concurrent diagnosis of Klinefelter's syndrome was made on the features of MR, increased height, and lack of secondary sexual characteristics.

His sister, IV 2 , had an onset of Parkinsonian features from age 19 , with bilateral hand tremor and speech impairment. She is now 22 and has minimal dysarthria and upper limb rigidity but no tremor on treatment with low dose Pergolide and Sinement, although higher dose leads to involuntary movements with hyperactivity.

Patient III. 3 is in her fifth decade and has no known symptoms to date. She has three children, all also asymptomatic.

Patient IV.3 is 18 years old and has no symptoms or signs of Parkinsonism on examination.

Patient II.3 died of "Alzheimer disease" but no definite details are available. Both I 1 and I·2 lived to apparent old age and had no recorded neurological problems.

Patient II $\cdot 1$, spouse of II $\cdot 2$, is alive at the age of 84 , but has a seven year history of apparently coincidental arteriosclerotic Parkinsonism secondary to cerebral arteriosclerotic changes.

DNA has been stored on all available family members. There was no evidence clinically of dementia, cerebellar signs, or sensory nerve involvement. Radiological imaging was not indicated clinically as the family conformed to all the diagnostic clinical criteria for Parkinson's disease $^{2}$ and the diagnosis of PD was made independently by two different neurologists and confirmed by a clinical neurogeneticist. None of the affected cases had autonomic dysfunction, and all were dopa responsive with an estimated $80 \%$ response; tremor was completely abolished on treatment but very high doses induced abnormal movements and restlessness. Other conditions, such as Huntington's disease and chromosomal abnormalities, have been excluded on DNA and cytogenetic analysis, with the exception of IV 1 who had an abnormal male karyotype of $47, \mathrm{XXY}$ with no evidence of mosaicism. There was no additional evidence of Wilson's disease or abnormal copper metabolism, or other evidence of drug induced illness.

\section{Discussion}

Previous case reports of familial Parkinsonism have had the limitation that clinical details have been poor and several families have had atypical features. ${ }^{2}$ Using the criteria described by $\mathrm{Ma}$ raganore $e t a l,{ }^{2}$ who described 20 families with Parkinson's disease, our own family is typical of true familial PD, and when examined individually, appear clinically indistinguishable from sporadic PD cases. This finding, combined with previous case reports, shows that autosomal dominant PD families do exist, and are more common than previously thought, although because of the differing features, there is probably heterogeneity, as several families do not conform to set criteria for $\mathrm{PD},{ }^{2}$ and of those that do, most do not show anticipation. This suggests that although there is definitely familial PD, there is also familial Parkinsonism, and within PD there are different clinical types which may or may not have the same genetic cause. The unusual feature in our family is anticipation. This feature is often not noted or may be ascribed to ascertainment bias or to earlier diagnosis in a familial setting. In our own family, this is not the case, as the severity of the onset symptoms in all of the cases excludes this. The anticipation is also unusually severe; the index patient in generation II had an onset of PD at 68 years. Patient IV 1 had an onset at 14 years, a difference in two generations of 54 years. If anticipation in PD is shown to be the result of an expanded trinucleotide repeat, it is possible that a mechanism such as in myotonic dystrophy could be responsible. ${ }^{12}$ The clinical similarity to the inheritance in myotonic dystrophy with increased anticipation through maternal transmission in this family makes a CTG or other combination of nucleotides, such as CGG or CCA, possible contenders, unlike most late onset repeat diseases which show paternally inherited anticipation with a CAG repeat. This makes the origin of a mutation in this family from I.1 more likely, although we cannot exclude the fact that the small size of this family may give a false impression of increased anticipation from affected females. The opposite occurs in most late onset neurogenetic diseases, where advanced anticipation usually occurs with transmission from the affected father. Carrero-Valenzuela $e t$ al $^{13}$ recently analysed 46 unrelated PD probands for CAG expansion. Their results showed that $37 \%$ had expanded CAG repeats, a level higher (but not significantly so) than the control population. This could suggest that most familial PD without anticipation does not have a CAG repeat basis. Two of their cases, however, had large CAG repeats, although both parental samples were not available to confirm familial transmission. This strengthens our hypothesis above that $P D$ is heterogeneous with different genetic causes. There may still be a CAG or other triplet involvement in some rare forms of PD. We cannot state if our family is similar to all others, as detailed published descriptions are rare; however, our family appears clinically similar to a large Greek-American family, ${ }^{6}$ which shows anticipation in three generations. Clearly more molecular analyses need to be carried out on a much larger PD sample with very carefully chosen population controls. This will hopefully resolve whether CAG expansions exist in all PD or in some rare PD families, if another trinucleotide repeat is present, or if some alternative underlying mechanism is responsible. Anticipation has been clearly shown in PD by Payami et al, ${ }^{1}$ with an earlier age of onset of 17 years on average in an analysis of $137 \mathrm{PD}$ patients and families, so this effect is real. We tested the family for the presence of Huntington's disease (HD) as occasionally HD can be an atypical presentation $^{14}$; the result was negative. The 
coincidental presence of Klinefelter's syndrome in IV' 1 could account for some accentuation of the tremor, ${ }^{15}$ and the subsequent earlier recognition and diagnosis of PD. However, the effect is small, and his affected sister also shows marked anticipation. There was no evidence of other neurological conditions segregating in this family. The exact cause and relevance of the "dementia" illness in II. 3 is unknown. Nigrostriatal damage also occurs in amyotrophic lateral sclerosis, although recent evidence has excluded SOD-1 mutations in patients with familial PD. ${ }^{16}$

In conclusion, this family shows three living generations of affected PD patients. The findings display autosomal dominant inheritance with strong evidence of severe anticipation. This is suggestive of involvement of a trinucleotide or similar expansion phenomenon in the causal process. PD is probably genetically heterogeneous with different genetic and environmental influences, but at least a small proportion is definitively autosomal dominant with anticipation, and families like this may have a closely similar genetic aetiology. It is hoped that identification and molecular investigation of such families will lead to an increased understanding of Parkinsonism.

1 Payami H, Larsen K, Bernard S, Nutt J. Increased risk of Parkinson's disease in parents and siblings of patients. Ann Neurol 1994;36:659-61.
2 Maraganore DM, Harding AE, Marsden CD. A clinical and genetic study of familial Parkinson's disease. Mov Disord 1991;6:205-11.

3 Harding AE. Inherited movement disorders. In: Harding $\mathrm{AE}$, ed. Genetics in neurology. London: Baillière Tindall, 1994:259-80.

4 Golbe LI, Di Iorio G, Bonavita V, Miller DC, Duvoisin RC. A large kindred with autosomal dominant Parkinson's disease. Ann Neurol 1990;27:276-82.

5 Payami H, Bernard S, Larsen K, Kaye J, Nutt J. Genetic anticipation in Parkinson's disease. Neurology 1995;45: $135-8$.

6 Markopoulou K, Wszolek ZK, Pfeiffer RF. A GreekAmerican kindred with autosomal dominant levodoparesponsive parkinsonism and anticipation. Ann Neurol 1995;38:373-8.

7 Bonifati V, Vanacore N, Meco G. Anticipation of onset age in familial Parkinson's disease. Neurology 1994;44:1978-9.

8 Golbe LI, Di Iorio G, Lazzarini AM, et al. A large kindred with Parkinson's disease (PD): onset age, segregation ratios and anticipation. Mov Disord 1993;8:406A.

9 Morrison PJ. Trinucleotide repeat repeat repeat. Lancet 1993;342:385-6.

10 O'Donovan MC, Guy C, Craddock, et al. Expanded CAG repeats in schizophrenia and bipolar disorder. Nature Genet 1995;10:379-80.

11 MacMillan JC, Morrison PJ, Nevin NC, et al. Identification of an expanded CAG repeat in the Huntington's disease of an expanded CAG repeat in the Huntington's disease
gene (IT15) in a family reported to have benign hereditary gene (IT15) in a family reported to have
chorea. $\mathcal{F}$ Med Genet 1993;30:1012-13.

12 Harley HG, Rundle SA, MacMillan JC, et al. Size of the unstable CTG repeat sequence in relation to phenotyp and parental transmission in myotonic dystrophy. $A m$ Hum Genet 1993;52:1164-74.

13 Carrero-Valenzuela $\mathrm{R}$, Lindblad $\mathrm{K}$, Payami $\mathrm{H}$, et al. No evidence for association of familial Parkinson's diseas with CAG repeat expansion. Neurology 1995;45:1760-3.

14 Morrison PJ, Johnston WP, Nevin NC. The epidemiology of Huntington's disease in Northern Ireland. 7 Med Genet 1995;32:524-30.

15 Gorlin RJ, Cohen MM Jr, Levin LS, eds. Syndromes of the head and neck. Oxford monographs on medical genetics No 19. Oxford: Oxford University Press, 1990.

16 Bandman $\mathrm{O}$, Davis MB Marsden CD, Harding AE. Sequence of the superoxide dismutase 1 (SOD 1) gene in familial Parkinson's disease. $\mathcal{F}$ Neurol Neurosurg Psychiatry 1995;59:90-1. 